\title{
Erratum
}

\section{Erratum: Liesz et al., "DAMP Signaling Is a Key Pathway Inducing Immune Modulation after Brain Injury"}

Erratum notice for "DAMP Signaling Is a Key Pathway Inducing Immune Modulation after Brain Injury", by Arthur Liesz, Alexander Dalpke, Eva Mracsko, Daniel J. Antoine, Stefan Roth, Wei Zhou, Huan Yang, Shin-Young Na, Mustafa Akhisaroglu, Thomas Fleming, Tatjana Eigenbrod, Peter P. Nawroth, Kevin J. Tracey, and Roland Veltkamp, which appeared on pages 583-598 of the January 14, 2015 issue.

The corresponding authors and editors received notice from the University of Liverpool about allegations of research misconduct by Daniel Antoine. These investigations indicated that Dr. Antoine, a co-author on this article, may have fabricated mass spectrometry data. Dr. Peter Clegg from University of Liverpool, who led the investigation into the misconduct, states: "I have identified similar data fabrication in a large number of papers co-authored by Dr. Antoine and would like to advise that any mass spectrometry data generated by Dr. Antoine should be considered as unreliable and potentially fraudulent.” Dr. Antoine left employment at the University of Liverpool in September 2017 and could not be reached by university officials nor the co-authors of this paper. Therefore, the authors and editors decided to remove this potentially fraudulent mass spectrometry dataset provided by Dr. Antoine from the abovementioned article.

The authors state, "Neither the corresponding author of this manuscript, Roland Veltkamp and Arthur Liesz, nor any other co-author of this manuscript have been involved in the fraudulent data set presented in Figure 2, which was contributed solely by Dr. Antoine. We would like to emphasize that the representative mass spectra in Figure 2 and results on acetylated HMGB1 are only ancillary and do not contribute substantially to the overall conclusions of this manuscript. Also, Dr. Antoine was neither involved in experiments nor contributed data to the other results of this manuscript, including those presented in the six result figures (Figs. 1, 3-7), which present data from experimental stroke models and immunological readouts. The main conclusion of our results are that alarmins released from necrotic brain tissue induce a sterile inflammatory immune response via pattern-recognition receptors. This conclusion is not based on the mass spec data contributed by Dr. Antoine and would not be affected by removing this data set. Moreover, key findings of this manuscript and the main conclusion have been meanwhile reproduced several times by independent investigators in our laboratories. Removing Dr. Antoine from the author list and the erroneous results on HMGB1 acetylation from Figure 2 would not change the conclusion of the manuscript in any way and would still leave the otherwise important data set of this manuscript available for the scientific community."

The online version of the article has been updated to remove reference to and discussion of the questionable data as follows:

- Figure 2 and its legend have been removed.

- On page 583, line 4 of the abstract, "We analyzed release of HMGB1 isoforms and investigated its inflammatory potency and signaling pathways..." now reads "We investigated the inflammatory potency of HMGB1 and its signaling pathways. .."

- On page 583, line 7 of the abstract, the sentence "Our results indicate that the cytokine-inducing, fully reduced isoform of HMGB1 was released from the ischemic brain in the hyperacute phase of stroke in mice and patients." has been edited to remove "the cytokine-inducing, fully reduced isoform of".

- On page 586, the entire section titled “Analysis of HMGB1 by electrospray ionization liquid chromatography mass spectrometry” has been removed.

- On page 588, the entire section titled "HMGB1 matures in the circulation to its cytokine-inducing isoform" has been removed.

- In the original Figure 8 legend, line 2, "(fully reduced, hypoacetylated)" has been removed.

- The author line and contributions have been updated to remove Daniel Antoine.

DOI: 10.1523/JNEUROSCI.0878-19.2019 